Supporting information:

\title{
Efficient And Simple Colorimetric Fluoride Ion Sensor Based On Receptors Having Urea And Thiourea
} Binding Sites.

D. Amilan Jose, D. Krishna Kumar, Bishwajit Ganguly* and Amitava Das*

Central Salt and Marine Chemicals Research Institute, Bhavnagar: 364002, Gujarat, India. E-Mail: amitavaad1@sancharnet.in, salt@csir.res.in 


\section{$\underline{\text { Content }}$}

1. Synthesis

2. Uv-vis spectra of receptors 1 and 2 with different anions.

3. Spectral changes for receptor 1 in presence of Fluoride ion at different temperatures $\left(25-70^{\circ} \mathrm{C}\right)$.

4. UV-vis titration curves (Job Plots).

5. Color change for the receptor $\mathbf{1}$ and $\mathbf{2}$.

6. Cartesian Coordinates for receptor 1 and 2 and their corresponding complexes with halides.

7. Equation used for evaluation of K.

8. Discussion on results of electrochemical studies. 


\section{Synthesis.}

\section{Receptor 1}

1,2- diaminoanthraquinone (200mg, 0.839mmol) was dissolved in a solvent mixture of dry DMF and THF (1:4 v/v) .To this solution was added phenylisocyanate (199.5mg $1.678 \mathrm{mmol})$ and stirred under inert atmosphere at $90^{\circ} \mathrm{C}$ for 8 hrs. The reaction mixture was cooled to room temperature. Precipitate formed was filtered and washed thoroughly with THF and acetonitrile. Yield 60\%.

Elemental analysis; Calculated for $\mathrm{C}_{28} \mathrm{H}_{20} \mathrm{~N}_{4} \mathrm{O}_{4}$ : C 70.58, $\mathrm{H}$ 4.20, N 11.76; Experimental: C 69.9, H 4.5, N 12.1.

${ }^{1}$ H NMR (200 MHz, DMSO-d 6 , ppm): 9.795 (1H,-NH), 9.719 (1H,-NH), 9.11 $(1 \mathrm{H}, \mathrm{s}, \mathrm{NH}), \quad 8.67 \quad(1 \mathrm{H}, \mathrm{d}, 8.6 \mathrm{~Hz}), \quad 8.624(1 \mathrm{H}, \mathrm{s},-\mathrm{NH}), \quad 8.137-8.181(3 \mathrm{H}, \mathrm{m})$, 7.942(m,2H), 7.478-7.584(m,4H), 7.28-7.35(m,4H), 6.98-7.049(m,2H).

FT-IR (KBr) $\mathrm{cm}^{-1}$ : 3366(-NH), 3249(-NH), 3062, 1735, 1660 (C=O), 1594, 1553, 1444, 1333, 1280, 1248, 1216, 748, 715,693.

Uv-Vis $\left(\mathrm{CH}_{3} \mathrm{CN} / \mathrm{DMSO}, 9: 1, \mathrm{v} / \mathrm{v}\right): \lambda_{\max }(\mathrm{nm})\left(\varepsilon \mathrm{M}^{-1} \mathrm{dm}^{3} \mathrm{~cm}^{-1}\right): 422(8160)$.

\section{Receptor 2}

The procedure followed was for same as receptor 1; except phenylisothiocyanate (226.5mg 1.678mmol) was used instead of phenylisocyanate. yield 82\% 
Elemental analysis Calculated for $\mathrm{C}_{28} \mathrm{H}_{20} \mathrm{~N}_{4} \mathrm{O}_{2} \mathrm{~S}_{2:} \mathrm{C}$ 66.14, H 3.93, N 11.02,S 12.59 Experimental: C 65.8, H 3.6, N 10.9, S 12.3

${ }^{1} \mathrm{H}$ NMR (200 MHz, DMSO-d 6 , ppm): 13.252(2H, brS, $-2 \mathrm{NH}$ ), 12.640(2H, brS, 2NH), 8.178-8.219(6H,m), 7.956-8.038(4H,m), 7.912-7.940(4H,m), 7.521-7.562 $(2 \mathrm{H}, \mathrm{d}, 8.2 \mathrm{~Hz})$.

FT-IR (KBr) cm ${ }^{-1}: 3247$ (-NH), 3191 (-NH), 1664 (C=S), 1589, 1497, 1383, 1330, 1288, 1150, 842, 713, 575, 485.

Uv-Vis $\left(\mathrm{CH}_{3} \mathrm{CN} / \mathrm{DMSO}, 9: 1, \mathrm{~V} / \mathrm{V}\right): \lambda_{\max }(\mathrm{nm})\left(\varepsilon \mathrm{M}^{-1} \mathrm{dm}^{3} \mathrm{~cm}^{-1}\right): 459$ (18680). 


\section{Uv-vis spectra of receptors 1 and 2 with different anions.}

\section{Receptor 1.}

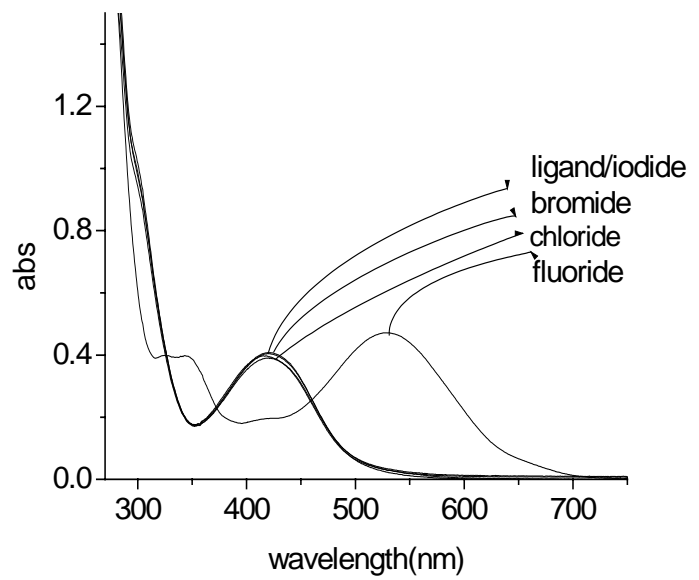

SI Figure 1: $[1]=5.0 \times 10^{-5} \mathrm{M},\left[\mathrm{X}^{-}\right]=5.0 \times 10^{-3} \mathrm{M}$, solvent used: $\mathrm{DMSO} / \mathrm{CH}_{3} \mathrm{CN}(1: 9$, $\mathrm{v} / \mathrm{v})$.

$\underline{\text { Receptor } 2}$

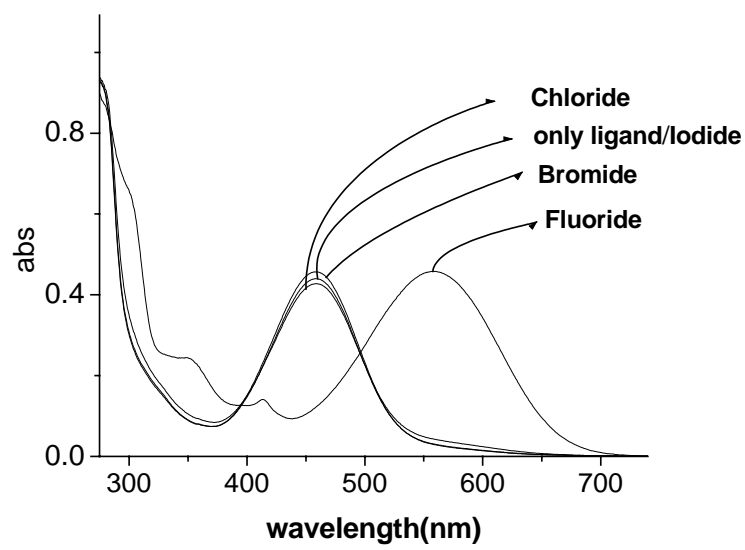

SI Figure 2: $[2]=2.5 \times 10^{-5} \mathrm{M},\left[\mathrm{X}^{-}\right]=2.5 \times 10^{-3} \mathrm{M}$, solvent used: $\mathrm{DMSO} / \mathrm{CH}_{3} \mathrm{CN}(1: 9$, $\mathrm{v} / \mathrm{v})$. 
3. Spectral changes for receptor 1 in presence of Fluoride ion at different temperatures $\left(25-70^{\circ} \mathrm{C}\right)$.

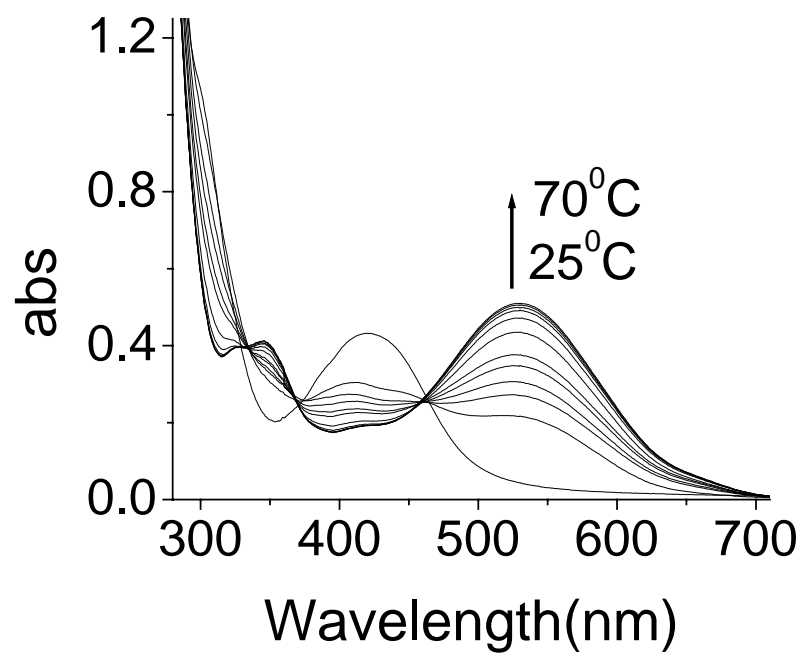

SI Figure 3: $[1]=5.0 \times 10^{-5} \mathrm{M},\left[\mathrm{F}^{-}\right]=4.0 \times 10^{-4} \mathrm{M}$, Temperature was raised from $25^{\circ} \mathrm{C}$ to $70^{\circ} \mathrm{C}$, solvent used: $\mathrm{DMSO} / \mathrm{CH}_{3} \mathrm{CN}(1: 9, \mathrm{v} / \mathrm{v})$. 


\section{4. $\underline{\mathrm{UV} \text {-vis titration curves }}$}

Effect of fluoride ion
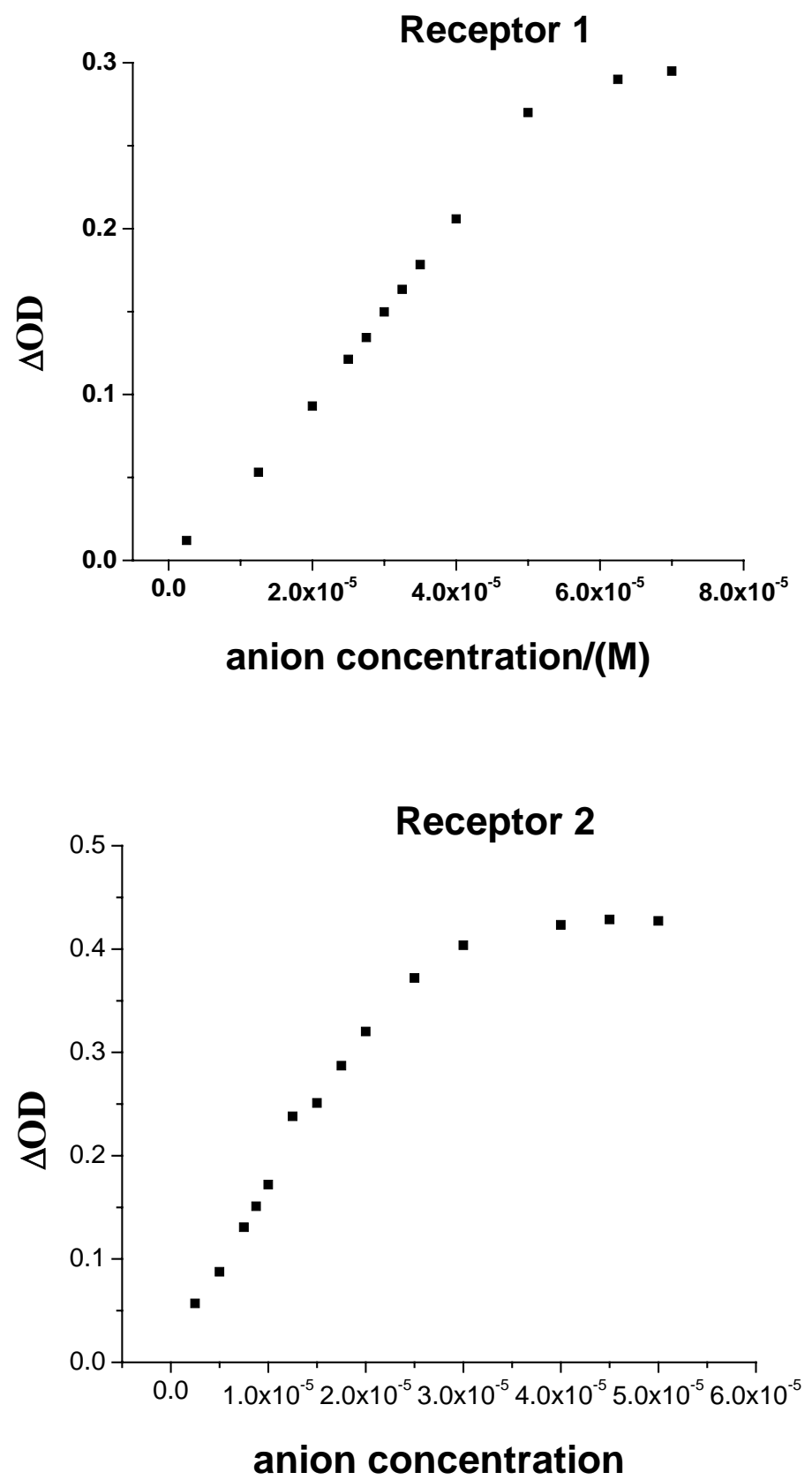

$\Delta \mathrm{OD}=$ change in absorbance at 530nm (for receptor 1 ) and 561nm (for receptor2). 


\section{Color change}

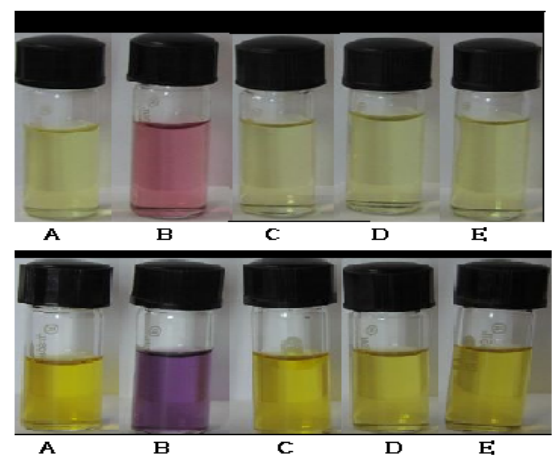

Receptor 1

Receptor 2

$\mathrm{A}=$ Free Receptor, $\mathrm{B}=$ with Fluoride, $\mathrm{C}=$ with Chloride, $\mathrm{D}=$ with Bromide, $\mathrm{E}=$ with lodide Anions added 5 equivalent 


\section{RHF/6-31G* optimized Cartesian coordinates for receptors 1 and 2 and their corresponding complexes with $\mathrm{F}-$, $\mathrm{Cl}$ - and $\mathrm{Br}-$.}

Receptor 1: B3LYP/6-31G*//RHF/6-31G* (total energy) $=-1445.33836$ Hartree

\begin{tabular}{|c|c|c|c|}
\hline Atomic number & $\mathrm{x}$ & $\mathrm{y}$ & $\mathrm{z}$ \\
\hline 6 & 1.251995 & -1.042111 & -.302552 \\
\hline 6 & 1.428778 & .152568 & -1.015270 \\
\hline 6 & 3.214925 & -1.967157 & -1.344555 \\
\hline 6 & 2.526275 & .281103 & -1.881266 \\
\hline 6 & 2.142974 & -2.089334 & -.486368 \\
\hline 6 & 3.411300 & -.792471 & -2.042568 \\
\hline 6 & 2.829125 & 1.556271 & -2.602435 \\
\hline 1 & 1.996493 & -2.992676 & .068696 \\
\hline 1 & 3.900112 & -2.780076 & -1.483310 \\
\hline 6 & 3.925306 & 1.551904 & -3.606806 \\
\hline 6 & 4.730913 & .519992 & -3.779081 \\
\hline 6 & 4.565160 & -.724573 & -2.988063 \\
\hline 8 & 5.329063 & -1.632723 & -3.125988 \\
\hline 7 & .568073 & 1.254859 & -.814488 \\
\hline 6 & -.785982 & 1.244346 & -1.034192 \\
\hline 7 & -1.322767 & 2.492661 & -1.075568 \\
\hline 6 & -2.692082 & 2.830318 & -1.180176 \\
\hline 6 & -5.341274 & 3.661882 & -1.381215 \\
\hline 6 & -3.618831 & 2.036320 & -1.844065 \\
\hline 6 & -3.094846 & 4.039014 & -.625346 \\
\hline 6 & -4.410215 & 4.453885 & -.731155 \\
\hline 6 & -4.935778 & 2.456968 & -1.930318 \\
\hline 1 & -3.318106 & 1.105188 & -2.276074 \\
\hline 1 & -4.704420 & 5.393001 & - . 298061 \\
\hline 1 & -5.647563 & 1.834455 & -2.442410 \\
\hline 1 & -6.365261 & 3.978845 & -1.459880 \\
\hline 7 & .168090 & -1.175061 & .574157 \\
\hline 6 & .236325 & -1.751767 & 1.815253 \\
\hline 7 & -.978138 & -1.761559 & 2.443058 \\
\hline 6 & -1.306092 & -2.272540 & 3.713446 \\
\hline 6 & -2.144874 & -3.227482 & 6.198877 \\
\hline 6 & -.377651 & -2.796819 & 4.606502 \\
\hline 6 & -2.650469 & -2.229939 & 4.074965 \\
\hline 6 & -3.064031 & -2.702133 & 5.305844 \\
\hline 6 & -.810128 & -3.268347 & 5.836187 \\
\hline 1 & .657140 & -2.835935 & 4.343794 \\
\hline 1 & -4.107412 & -2.658931 & 5.563078 \\
\hline 1 & - . . 082425 & -3.672184 & 6.517619 \\
\hline 1 & -2.463228 & -3.596777 & 7.156786 \\
\hline 1 & 4.034067 & 2.471062 & -4.151788 \\
\hline 1 & 5.543318 & .527625 & -4.481760 \\
\hline 1 & -.712666 & 3.256365 & - . 890998 \\
\hline 1 & -.703652 & -.848480 & .216512 \\
\hline 1 & -1.744620 & -1.373931 & 1.941670 \\
\hline 1 & 1.018438 & 2.120409 & -1.013709 \\
\hline 8 & 2.258388 & 2.595204 & -2.397624 \\
\hline 1 & -3.376033 & -1.826470 & 3.388844 \\
\hline 1 & -2.379789 & 4.653905 & - . 106061 \\
\hline 8 & -1.434651 & .232576 & -1.154647 \\
\hline 8 & 1.250183 & -2.188943 & 2.286143 \\
\hline
\end{tabular}


Fluoride complexed receptor 1: $\mathrm{B} 3 \mathrm{LYP} / 6-31 \mathrm{G} * / / \mathrm{RHF} / 6-31 \mathrm{G} *$ (total energy) $=$ -1545.28393 Hartree

\begin{tabular}{|c|c|c|c|c|}
\hline Atomic $r$ & number & $x$ & $\mathrm{y}$ & z \\
\hline & 6 & 1.460756 & -.936848 & - . 241193 \\
\hline & 6 & 1.309602 & .293298 & -.926099 \\
\hline & 6 & 3.258886 & -1.525290 & -1.716284 \\
\hline & 6 & 2.164055 & .606664 & -1.972067 \\
\hline & 6 & $2.4550 \odot 2$ & -1.824787 & -.634998 \\
\hline & 6 & 3.118389 & -.331612 & -2.399548 \\
\hline & 6 & 2.183029 & 1.961892 & -2.616155 \\
\hline & 1 & 2.564705 & -2.748816 & - .108783 \\
\hline & 1 & 3. 998291 & -2.228284 & -2.049087 \\
\hline & 6 & 2.845998 & 2.057260 & -3.951443 \\
\hline & 6 & 3.676281 & 1.130276 & -4.393882 \\
\hline & 6 & 3.948865 & -.100257 & -3.607430 \\
\hline & 8 & 4.796463 & -.867863 & -3.974290 \\
\hline & 7 & .263618 & 1.139322 & -.524751 \\
\hline & 6 & - . 798823 & 1.425259 & -1.351438 \\
\hline & 7 & -1.909424 & 1.741652 & - . 621400 \\
\hline & 6 & -3.157065 & 2.128823 & -1.104496 \\
\hline & 6 & -5.726032 & 2.935264 & -1.875122 \\
\hline & 6 & -3.390072 & 2.596479 & -2.396996 \\
\hline & 6 & -4.225357 & 2.072308 & - . 206986 \\
\hline & 6 & -5.490790 & 2.471699 & -.589925 \\
\hline & 6 & -4.666378 & 2.990115 & -2.764706 \\
\hline & 1 & -2.584406 & 2.637207 & -3.098877 \\
\hline & 1 & -6.297015 & 2.416951 & .121474 \\
\hline & 1 & -4.828018 & 3.346722 & -3.767814 \\
\hline & 1 & -6.711854 & 3.244455 & -2.175391 \\
\hline & 7 & .573890 & -1.195029 & .793510 \\
\hline & 6 & .768583 & -2.008067 & 1.882587 \\
\hline & 7 & -.138607 & -1.701701 & 2.856646 \\
\hline & 6 & - . 327885 & -2.325822 & 4.087369 \\
\hline & 6 & -.875807 & -3.442735 & 6.597497 \\
\hline & 6 & .348399 & -3.468472 & 4.512107 \\
\hline & 6 & -1.278853 & -1.752975 & 4.935952 \\
\hline & 6 & -1.546306 & -2.305660 & 6.172173 \\
\hline & 6 & .065956 & -4.009387 & 5.756768 \\
\hline & 1 & 1.083586 & -3.916251 & 3.878577 \\
\hline & 1 & -2.283670 & -1.843441 & 6.805797 \\
\hline & 1 & .598688 & -4.891877 & 6.067940 \\
\hline & 1 & -1.083065 & -3.874223 & 7.560943 \\
\hline & 1 & 2.662220 & 2.972616 & -4.483558 \\
\hline & 1 & 4.212761 & 1.222454 & -5.320803 \\
\hline & 1 & -1.870797 & 1.430056 & .341973 \\
\hline & 1 & -.193375 & - . 548011 & .920574 \\
\hline & 1 & -.686141 & - . 869275 & 2.677267 \\
\hline & 1 & .029674 & 1.141230 & .451943 \\
\hline & 8 & 1.802304 & 2.966870 & -2.100997 \\
\hline & 1 & -1.802114 & -.870745 & 4.611133 \\
\hline & 1 & -4.047799 & 1.708176 & .789754 \\
\hline & 8 & -.735633 & 1.386644 & -2.553486 \\
\hline & 8 & 1.613742 & -2.864756 & 1.960838 \\
\hline & 9 & -1.202847 & .554406 & 1.713141 \\
\hline
\end{tabular}


Chloride complexed receptor 1: $\mathrm{B} 3 \mathrm{LYP} / 6-31 \mathrm{G} * / / \mathrm{RHF} / 6-31 \mathrm{G}$ * (total energy)= -1905.66864 Hartree

\begin{tabular}{|c|c|c|c|c|}
\hline Atomic $\mathrm{n}$ & number & $x$ & $y$ & z \\
\hline 6 & 5 & 1.472637 & -1.058165 & - . 107930 \\
\hline 6 & 6 & 1.463046 & .188902 & -.770032 \\
\hline 6 & 6 & 3.326788 & -1.745149 & -1.468089 \\
\hline 6 & 6 & 2.417399 & .464083 & -1.740020 \\
\hline 6 & 6 & 2.428102 & -2.010884 & -.458421 \\
\hline 6 & 6 & 3.331715 & -.525740 & -2.122691 \\
\hline 6 & 6 & 2.564247 & 1.826800 & -2.354527 \\
\hline 1 & 1 & 2.432513 & -2.954951 & .039628 \\
\hline 1 & 1 & 4.037309 & -2.493248 & -1.763312 \\
\hline 6 & 6 & 3.357744 & 1.914727 & -3.615598 \\
\hline 6 & 6 & 4.161881 & .945407 & -4.012947 \\
\hline 6 & 6 & 4.275254 & -.324362 & -3.250343 \\
\hline 8 & 3 & 5.090811 & -1.144243 & -3.571206 \\
\hline 7 & 7 & .441271 & 1.098949 & - . .441998 \\
\hline 6 & 6 & -.479384 & 1.545497 & -1.354113 \\
\hline 7 & 7 & -1.480454 & 2.260053 & - . .763378 \\
\hline 6 & 6 & -2.584388 & 2.849469 & -1.389465 \\
\hline 6 & 6 & -4.841905 & 4.104071 & -2.460933 \\
\hline 6 & 6 & -2.640629 & 3.150410 & -2.748103 \\
\hline 6 & 6 & -3.666662 & 3.184677 & -.575771 \\
\hline 6 & 6 & -4.779438 & 3.806386 & -1.108698 \\
\hline 6 & 6 & -3.766900 & 3.769043 & -3.266153 \\
\hline 1 & 1 & -1.820900 & 2.892676 & -3.384029 \\
\hline 1 & 1 & -5.602369 & 4.054432 & -.460909 \\
\hline 1 & 1 & -3.795966 & 3.992282 & -4.318991 \\
\hline 1 & 1 & -5.709758 & 4.585024 & -2.876869 \\
\hline 7 & 7 & .515727 & -1.259286 & .877129 \\
\hline 6 & 6 & .369930 & -2.340017 & 1.717763 \\
\hline 7 & 7 & -.571564 & -2.100481 & 2.671304 \\
\hline 6 & 6 & -1.006305 & -2.958689 & 3.689115 \\
\hline 6 & 6 & -2.015012 & -4.515042 & 5.782024 \\
\hline 6 & 6 & -.665541 & -4.305048 & 3.787958 \\
\hline 6 & 6 & -1.856492 & -2.401916 & 4.646391 \\
\hline 6 & 6 & -2.352764 & -3.174005 & 5.677598 \\
\hline 6 & 6 & -1.172550 & -5.063701 & 4.832188 \\
\hline 1 & 1 & - . .012673 & -4.745274 & 3.065721 \\
\hline 1 & 1 & -3.006146 & -2.721810 & 6.403346 \\
\hline 1 & 1 & -.898457 & -6.102922 & 4.895000 \\
\hline 1 & 1 & -2.400647 & -5.116400 & 6.586366 \\
\hline 1 & 1 & 3.284065 & 2.855099 & -4.130241 \\
\hline 1 & 1 & 4.786208 & 1.026600 & -4.884029 \\
\hline 1 & 1 & -1.552430 & 2.168380 & .232602 \\
\hline 1 & 1 & -.102725 & - . 493218 & 1.049016 \\
\hline 1 & 1 & -.931834 & -1.164682 & 2.722580 \\
\hline 1 & 1 & .348316 & 1.402873 & .505949 \\
\hline 8 & 3 & 2.171564 & 2.837690 & -1.859178 \\
\hline 1 & 1 & -2.120394 & -1.361670 & 4.572303 \\
\hline 1 & 1 & -3.627581 & 2.948795 & .473157 \\
\hline 8 & $B$ & - . 396382 & 1.312679 & -2.532426 \\
\hline 8 & 8 & .993784 & -3.364877 & 1.612013 \\
\hline 17 & & -1.327308 & 1.184876 & 2.453086 \\
\hline
\end{tabular}


Bromide complexed receptor 1: $\mathrm{B} 3 \mathrm{LYP} / 6-31 \mathrm{G} * / / \mathrm{RHF} / 6-31 \mathrm{G} *$ (total energy)= -4017.18308 Hartree

Atomic number

6

6

1

6

6

6

8

7

6

7

6

6

6

6

6

6

1

1

1

1

\begin{tabular}{|c|c|c|}
\hline$x$ & $y$ & $z$ \\
\hline 1.560681 & -1.195061 & -.201333 \\
\hline 1.554772 & - .046563 & -1.022054 \\
\hline 3.340938 & -2.109685 & -1.524676 \\
\hline 2.472602 & .068470 & -2.057442 \\
\hline 2.478110 & -2.213474 & -.456121 \\
\hline 3.347518 & - . 988801 & -2.337058 \\
\hline 2.616882 & 1.329193 & -2.861549 \\
\hline 2.479652 & -3.083532 & .162348 \\
\hline 4.022089 & -2.909757 & -1.742755 \\
\hline 3.366458 & 1.225843 & -4.148025 \\
\hline 35195 & .191753 & -4.437551 \\
\hline 4.250072 & -.966623 & -3.514902 \\
\hline 5.033915 & -1.844170 & -3.750852 \\
\hline .568770 & .928495 & - . 780039 \\
\hline 8338 & 1.248024 & -1.699012 \\
\hline 893 & 2.056735 & -1.168408 \\
\hline 3175 & 2.532171 & -1.802855 \\
\hline 4.859254 & 3.582891 & -2.896253 \\
\hline $9 \odot 99$ & 2.651001 & -3.183090 \\
\hline 3.559455 & 2.943721 & - . 977387 \\
\hline-4.716727 & 3.465614 & -1.522330 \\
\hline 710 & 70272 & -3.712117 \\
\hline-1.85 & 2.330926 & -3.825048 \\
\hline 5.511670 & 3.776596 & - . 866853 \\
\hline-3.912379 & 3.253583 & -4.781508 \\
\hline-5.762070 & 3.985626 & -3.320820 \\
\hline .641977 & -1.234995 & .838363 \\
\hline 994 & -2.2 & 1.788756 \\
\hline-420601 & -1.824888 & 2.742865 \\
\hline - . . 861957 & -2.539434 & 3.863550 \\
\hline-1.872481 & -3.789374 & 6.149407 \\
\hline-.592836 & -3.884074 & 4.101350 \\
\hline-1.64 & -1.8 & 4.778257 \\
\hline-2.1 & -2.4 & 5598 \\
\hline-1.1 & -4.490224 & 5.240914 \\
\hline .005139 & -4.439579 & 3.411499 \\
\hline-2.736442 & -1.881901 & 6.596819 \\
\hline-.882959 & -5.530639 & 5.412220 \\
\hline-2.259386 & -4.273484 & 7.028751 \\
\hline 3.2 & 2.0895 & -4.783000 \\
\hline 4.72 & .139090 & -5.332524 \\
\hline-1.376245 & 2.113865 & -.167655 \\
\hline .077297 & -.418605 & .951316 \\
\hline-.725196 & -.869357 & 2.708932 \\
\hline & 1.383966 & . 110019 \\
\hline 2.253064 & 2.406087 & -2.501847 \\
\hline 1.846554 & - .788523 & 4.592787 \\
\hline-3.454288 & 2.842264 & .088622 \\
\hline & .839681 & - 2.831180 \\
\hline $02014+$ & -3.283508 & 1.766764 \\
\hline. .010112 & 1.591805 & 2.286281 \\
\hline
\end{tabular}


Receptor 2: B3LYP/6-31G*//RHF/6-31G* (total energy) $=-2091.22013$ Hartree

\begin{tabular}{|c|c|c|c|}
\hline Atomic & number & $\mathrm{y}$ & Z \\
\hline 6 & 1.160585 & -1.278874 & -.642683 \\
\hline 6 & 1.374052 & .051585 & -.990831 \\
\hline 6 & 3.071183 & -1.947512 & -1.931234 \\
\hline 6 & 2.471935 & .388074 & -1.792603 \\
\hline 6 & 2.002670 & -2.267432 & -1.121176 \\
\hline 6 & 3.306702 & -.626959 & -2.268719 \\
\hline 6 & 2.814046 & 1.808593 & -2.117340 \\
\hline 1 & 1.812643 & -3.284656 & -.839867 \\
\hline 1 & 3.725290 & -2.708772 & -2.308202 \\
\hline 6 & 3.893916 & 2.054061 & -3.109600 \\
\hline 6 & 4.655703 & 1.086040 & -3.586538 \\
\hline 6 & 4.453878 & -.327199 & -3.180622 \\
\hline 8 & 5.179591 & -1.183801 & -3.587092 \\
\hline 7 & .536318 & 1.067273 & -.467238 \\
\hline 6 & -.726553 & 1.351665 & -.866024 \\
\hline 7 & -1.167062 & 2.528197 & -.402153 \\
\hline 6 & -2.456004 & 3.105070 & -.628182 \\
\hline 6 & -4.910469 & 4.322267 & -.996555 \\
\hline 6 & -2.698543 & 3.824940 & -1.785666 \\
\hline 6 & -3.431601 & 2.993696 & .346799 \\
\hline 6 & -4.661085 & 3.604585 & .161523 \\
\hline 6 & -3.929391 & 4.430722 & -1.969801 \\
\hline 1 & -1.931526 & 3.898639 & -2.533941 \\
\hline 1 & -5.420018 & 3.517242 & .917710 \\
\hline 1 & -4.121063 & 4.985740 & -2.870086 \\
\hline 1 & -5.865357 & 4.794731 & -1.141091 \\
\hline 7 & .048776 & -1.631800 & .152771 \\
\hline 6 & .092724 & -1.990036 & 1.454984 \\
\hline 7 & -1.135468 & -2.194907 & 1.969520 \\
\hline 6 & -1.460029 & -2.618747 & 3.291399 \\
\hline 6 & -2.239787 & -3.425547 & 5.824033 \\
\hline 6 & -1.245793 & -1.784413 & 4.377165 \\
\hline 6 & -2.066050 & -3.850487 & 3.469255 \\
\hline 6 & -2.461388 & -4.251276 & 4.735920 \\
\hline 6 & -1.628034 & -2.194260 & 5.641655 \\
\hline 1 & -.772657 & - . 832943 & 4.228619 \\
\hline 1 & -2.932406 & -5.208427 & 4.869049 \\
\hline 1 & -1.452950 & -1.549719 & 6.484052 \\
\hline 1 & -2.539692 & -3.737763 & 6.808192 \\
\hline 1 & 4.034118 & 3.085611 & -3.374144 \\
\hline 1 & 5.458994 & 1.266000 & -4.276562 \\
\hline 1 & -.535493 & 3.077828 & .140694 \\
\hline 1 & - . .832090 & -1.377617 & - . 249293 \\
\hline 1 & -1.904593 & -2.153341 & 1.335635 \\
\hline 1 & 1.055938 & 1.851266 & -.137667 \\
\hline 8 & 2.290740 & 2.753926 & -1.594378 \\
\hline 1 & -2.220544 & -4.494015 & 2.621642 \\
\hline 1 & -3.228727 & 2.428777 & 1.238340 \\
\hline 16 & -1.666741 & .334020 & -1.827497 \\
\hline 16 & 1.518838 & -2.162285 & 2. 319836 \\
\hline
\end{tabular}


Fluoride complexed receptor 2: $\mathrm{B} 3 \mathrm{LYP} / 6-31 \mathrm{G} * / / \mathrm{RHF} / 6-31 \mathrm{G} *$ (total energy)= -2191.17790 Hartree

\begin{tabular}{|c|c|c|c|}
\hline Atomic number & $\mathrm{x}$ & $\mathrm{y}$ & z \\
\hline 6 & 1.486544 & -.969445 & -.315559 \\
\hline 6 & 1.289038 & .263197 & -.968758 \\
\hline 6 & 3.327710 & -1.430880 & -1.785608 \\
\hline 6 & 2.178296 & .674619 & -1.952417 \\
\hline 6 & 2.505411 & -1.811772 & -.747650 \\
\hline 6 & 3.180093 & -.195108 & -2.391536 \\
\hline 6 & 2.171335 & 2.069648 & -2.504889 \\
\hline 1 & 2.631873 & -2.762945 & $-.28 \odot 489$ \\
\hline 1 & 4.091654 & -2.095836 & -2.139589 \\
\hline 6 & 2.861648 & 2.272346 & -3.812172 \\
\hline 6 & 3.745560 & 1.407378 & -4.276276 \\
\hline 6 & 4.045208 & .145383 & -3.552275 \\
\hline 8 & 4.935979 & -.565516 & -3.926570 \\
\hline 7 & .165022 & 1.034711 & -.607877 \\
\hline 6 & -.922113 & 1.256532 & -1.390711 \\
\hline 7 & -1.974506 & 1.634439 & -.646580 \\
\hline 6 & -3.280318 & 1.964821 & -1.052867 \\
\hline 6 & -5.918737 & 2.649430 & -1.630162 \\
\hline 6 & -3.560758 & 2.843423 & -2.092264 \\
\hline 6 & -4.325085 & 1.446941 & -.295928 \\
\hline 6 & -5.635152 & 1.789828 & -.582609 \\
\hline 6 & -4.874412 & 3.170497 & -2.378537 \\
\hline 1 & -2.759434 & 3.256054 & -2.670373 \\
\hline 1 & -6.431927 & 1.378705 & .012486 \\
\hline 1 & $-5.08 \odot 478$ & 3.846474 & -3.190183 \\
\hline 1 & -6.936545 & 2.912951 & -1.859031 \\
\hline 7 & .591727 & -1.293755 & .713594 \\
\hline 6 & .797401 & -1.919719 & 1.907714 \\
\hline 7 & -.213461 & -1.641972 & 2.747039 \\
\hline 6 & -.426248 & -2.065444 & 4.073035 \\
\hline 6 & -1.053032 & -2.758109 & 6.699047 \\
\hline 6 & -.289214 & -3.381059 & 4.498831 \\
\hline 6 & -.896678 & -1.106286 & 4.963548 \\
\hline 6 & -1.209044 & -1.452020 & 6.266594 \\
\hline 6 & -.593660 & -3.714435 & 5.807244 \\
\hline 1 & .058428 & -4.129002 & 3.816672 \\
\hline 1 & -1.570633 & -.696482 & 6.942084 \\
\hline 1 & -.476954 & -4.735245 & 6.127288 \\
\hline 1 & -1.289399 & -3.028724 & 7.713225 \\
\hline 1 & 2.654569 & 3.208334 & -4.297120 \\
\hline 1 & 4.306948 & 1.574516 & -5.177409 \\
\hline 1 & -1.886149 & 1.370818 & .332770 \\
\hline 1 & -.228078 & -.711107 & .741713 \\
\hline 1 & -.781621 & -.842654 & 2.465320 \\
\hline 1 & - . . 027360 & 1.108430 & .377388 \\
\hline 8 & 1.758205 & 3.021721 & -1.918536 \\
\hline 1 & -1.018296 & -.094805 & 4.619029 \\
\hline 1 & -4.095926 & .778862 & .515213 \\
\hline 16 & -.937905 & 1.032112 & -3.066127 \\
\hline 16 & 2.127363 & -2.885183 & 2.293346 \\
\hline 9 & -1.261079 & .514289 & 1.635372 \\
\hline
\end{tabular}


Chloride complexed receptor 2: $\mathrm{B} 3 \mathrm{LYP} / 6-31 \mathrm{G} / / \mathrm{RHF} / 6-31 \mathrm{G} *$ (total energy)= -2551.55706 Hartree

\begin{tabular}{|c|c|c|c|}
\hline Atomic number & $x$ & $\mathrm{y}$ & z \\
\hline 6 & 1.766191 & -.816251 & - . .012085 \\
\hline 6 & 1.671649 & .343071 & -.800581 \\
\hline 6 & 3.686259 & -1.494227 & -1.279127 \\
\hline 6 & 2.606964 & .589660 & -1.798330 \\
\hline 6 & 2.804217 & -1.714392 & -.245491 \\
\hline 6 & 3.596081 & -.358435 & -2.067580 \\
\hline 6 & 2.624482 & 1.873358 & -2.577335 \\
\hline 1 & 2.895733 & -2.585655 & .363649 \\
\hline 1 & 4.465723 & -2.203550 & -1.480210 \\
\hline 6 & 3.426442 & 1.886691 & -3.835315 \\
\hline 6 & 4.321091 & .955652 & -4.113033 \\
\hline 6 & 4.536851 & -.202148 & -3.208390 \\
\hline 8 & 5.426435 & -.976222 & -3.424418 \\
\hline 7 & .615818 & 1.244536 & -.531044 \\
\hline 6 & -.518022 & 1.376070 & -1.249196 \\
\hline 7 & -1.404827 & 2.181992 & -.637388 \\
\hline 6 & -2.701070 & 2.549762 & -1.065744 \\
\hline 6 & -5.262868 & 3.387113 & -1.747699 \\
\hline 6 & -2.938614 & 3.112427 & -2.312664 \\
\hline 6 & -3.742860 & 2.422661 & -.157788 \\
\hline 6 & -5.017737 & 2.844560 & -.498450 \\
\hline 6 & -4.217720 & 3.516635 & -2.649869 \\
\hline 1 & -2.130523 & 3.221966 & -3.007496 \\
\hline 1 & -5.816780 & 2.741190 & .214418 \\
\hline 1 & -4.395126 & 3.944212 & -3.621186 \\
\hline 1 & -6.254031 & 3.708709 & -2.015824 \\
\hline 7 & .839603 & -.945143 & 1.026996 \\
\hline 6 & .343648 & -2.021326 & 1.688456 \\
\hline 7 & -.534618 & -1.636501 & 2.629609 \\
\hline 6 & -1.248611 & -2.439639 & 3.553470 \\
\hline 6 & -2.731424 & -3.845092 & 5.436860 \\
\hline 6 & -2.075907 & -3.478751 & 3.150201 \\
\hline 6 & -1.179087 & -2.096427 & 4.895326 \\
\hline 6 & -1.921905 & -2.795286 & 5.833296 \\
\hline 6 & -2.803463 & -4.181813 & 4.093553 \\
\hline 1 & -2.139941 & -3.732873 & 2.111377 \\
\hline 1 & -1.861777 & -2.518058 & 6.870949 \\
\hline 1 & -3.437369 & -4.990215 & 3.774276 \\
\hline 1 & -3.304908 & -4.392833 & 6.164011 \\
\hline 1 & 3.273955 & 2.748724 & -4.458209 \\
\hline 1 & 4.947590 & .992020 & -4.985422 \\
\hline 1 & -1.235217 & 2.338206 & .341091 \\
\hline 1 & .429221 & -.074244 & 1. 306584 \\
\hline 1 & -.639336 & -.645865 & 2.774296 \\
\hline 1 & .693192 & 1.798363 & .299928 \\
\hline 8 & 2.110712 & 2.887251 & -2.216509 \\
\hline 1 & -.548568 & -1.279337 & 5.196219 \\
\hline 1 & -3.547274 & 1.997520 & .810342 \\
\hline 16 & -.767724 & .588883 & -2.719765 \\
\hline 16 & .747550 & -3.632109 & 1.391668 \\
\hline 17 & -.478138 & 1.761040 & 2.594473 \\
\hline
\end{tabular}


Bromide complexed receptor 2: $\mathrm{B} 3 \mathrm{LYP} / 6-31 \mathrm{G} * / / \mathrm{RHF} / 6-31 \mathrm{G} *$ (total energy)= -4663.07303 Hartree

\begin{tabular}{|c|c|c|c|}
\hline Atomic number & $x$ & $y$ & z \\
\hline 6 & 1.800938 & -1.006342 & -.172187 \\
\hline 6 & 1.725776 & .057880 & -1.087087 \\
\hline 6 & 3.661254 & -1.896910 & -1.395189 \\
\hline 6 & 2.639113 & .150956 & -2.130198 \\
\hline 6 & 2.800067 & -1.963455 & -.322806 \\
\hline 6 & 3.588127 & -.858039 & -2.308590 \\
\hline 6 & 2.670380 & 1.328892 & -3.061044 \\
\hline 1 & 2.878739 & -2.760284 & .382405 \\
\hline 1 & 4.411013 & -2.652504 & -1.529487 \\
\hline 6 & 3.446375 & 1.168539 & -4.325251 \\
\hline 6 & 4.305021 & .181965 & -4.508992 \\
\hline 6 & 4.504383 & -.869869 & -3.480011 \\
\hline 8 & 5.361509 & -1.695676 & -3.622462 \\
\hline 7 & .711849 & 1.025964 & - . 898077 \\
\hline 6 & -.463086 & 1.077304 & -1.561541 \\
\hline 7 & -1.295924 & 1.987801 & -1.026193 \\
\hline 6 & -2.642973 & 2.266256 & -1.352764 \\
\hline 6 & -5.295222 & 2.947188 & -1.825403 \\
\hline 6 & -3.067290 & 2.538479 & -2.645665 \\
\hline 6 & -3.541296 & 2.352050 & -.296415 \\
\hline 6 & -4.860992 & 2.695356 & -.534912 \\
\hline 6 & -4.392463 & 2.866512 & -2.874075 \\
\hline 1 & -2.370730 & 2.484928 & -3.457208 \\
\hline 1 & -5.547435 & 2.758987 & .290937 \\
\hline 1 & -4.717122 & 3.068245 & -3.879945 \\
\hline 1 & -6.322494 & 3.206889 & -2.012524 \\
\hline 7 & .896854 & -.980179 & .895570 \\
\hline 6 & .346003 & -1.958545 & 1.659728 \\
\hline 7 & -.546298 & -1.439200 & 2.520820 \\
\hline 6 & -1.259131 & -2.059846 & 3.574719 \\
\hline 6 & -2.735394 & $-3.07 \odot 478$ & 5.703736 \\
\hline 6 & -1.978263 & -3.237211 & 3.422336 \\
\hline 6 & -1.300850 & -1.379273 & 4.785355 \\
\hline 6 & -2.038483 & -1.882199 & 5.843066 \\
\hline 6 & -2.701333 & -3.739595 & 4.490905 \\
\hline 1 & -1.967178 & -3.752025 & 2.484295 \\
\hline 1 & -2.063425 & -1.343730 & 6.773986 \\
\hline 1 & -3.250196 & -4.656550 & 4.366643 \\
\hline 1 & -3.304434 & -3.466695 & 6.526420 \\
\hline 1 & 3.306507 & 1.956417 & -5.042241 \\
\hline 1 & 4.912198 & .095550 & -5.391449 \\
\hline 1 & -1.049727 & 2.285273 & - . .098628 \\
\hline 1 & .547933 & - . .062431 & 1.094178 \\
\hline 1 & -.623988 & -.436424 & 2.531513 \\
\hline 1 & .848286 & 1.685268 & -.156570 \\
\hline 8 & 2.183940 & 2.390896 & -2.820920 \\
\hline 1 & - . .766184 & -.451374 & 4.882777 \\
\hline 1 & -3.191130 & 2.158027 & .701922 \\
\hline 16 & -.806435 & .085150 & -2.880173 \\
\hline 16 & .716249 & -3.600472 & 1.559445 \\
\hline 35 & -.293732 & 2.061356 & 2.290873 \\
\hline
\end{tabular}




\section{Equation used for evaluation of $\mathbf{K}$.}

$\mathrm{L}+\mathrm{A}^{-} \rightleftharpoons \mathrm{LA}^{-}$

Absorption maxima for $A^{-}$is $\lambda_{A^{-}}$, while extinction coefficient and $O D$ at this wavelength are $\varepsilon_{A^{-}}$and $A_{A^{-}}$respectively

Absorption maxima for $L^{-}{ }^{-}$is $\lambda_{L A^{-}}$, while extinction coefficient and $O D$ at this wavelength are $\varepsilon_{L A^{-}}$and $A_{L A^{-}}$respectively

- Thus, for a given concentration of the receptor molecule ([L]) and anion ([A- $]$ )

$\left[L A^{-}\right]=\left[\left\{A_{L A^{-}}-A^{0}\right\} / \varepsilon_{L A^{-}}\right]$

where $A^{0}$ is $O D$ value at $\lambda_{L A}$ - before addition of externally added $A^{-}$.

- $A_{L A^{-}}$was recorded at $\lambda_{L A^{-}}$after addition 100 mole equivalent of $A^{-}$.

Therefore, $\varepsilon_{\mathrm{LA}^{-}}=\mathrm{A}_{\mathrm{LA}}-/[\mathrm{L}]_{\text {free }}$

(assuming all the receptor molecules are bound to $\mathrm{A}^{-}$)

Thus, once we one can evaluate $\left[\mathrm{LA}^{-}\right]$, it is possible to calculate concentration of the uncomplexed recptor molecule, [L] using eq. 1 and 3.

- $[\mathrm{L}]_{\text {initial }}-\left[\mathrm{LA}^{-}\right]=[\mathrm{L}]_{\text {free }}$

---eq. 3

and, $\left[\mathrm{A}^{-}\right]_{\text {free }}=\left[\mathrm{A}^{-}\right]_{\text {initial }}-\left[\mathrm{LA}^{-}\right]$

---eq. 4

If $\mathrm{K}$ is formation constant for the inclusion complerx, $\mathrm{LA}^{-}$for a given concentration of $\mathrm{A}^{-}$ $K=\left[\mathrm{LA}^{-}\right] /\left\{\left[\mathrm{L}_{\text {free }}\left[\mathrm{A}^{-}\right]_{\text {free }}\right\}\right.$

---eq. 5 


\section{Discussion on results of electrochemical studies}

We also tried to evaluate the relative difference in the redox response of the quinone/semiquinone (q/sq) couple of the redox active anthraquinone moiety of the receptor 2 before and after fluoride ion binding, by electrochemical studies. Unlike the pyrrole-based derivative of anthraquinone, ${ }^{6 a}$ a small cathodic shift of $40 \mathrm{mV}$ was observed. The small change in $E_{1 / 2}$-values for the $\mathrm{q} / \mathrm{sq}$ couple is presumably due to complexity arising from the relative change in H-bonding and the nonplanarity of the quinone moiety before/ after binding with $\mathrm{F}^{-}$ ion, as it is observed in our calculation (vide infra). The small change in $E_{1 / 2}$-values for the q/sq couple value did not allow us to evaluate the association constant by electrochemical methods. 